\title{
XIII. On the morphological laws of the configurations formed by magnets floating vertically and subjected to the attraction of a superposed magnet; with notes on some of the phenomena in molecular structure which these experiments may serve to explain and illustrate
}

\section{Alfred M. Mayer}

To cite this article: Alfred M. Mayer (1879) XIII. On the morphological laws of the configurations formed by magnets floating vertically and subjected to the attraction of a superposed magnet; with notes on some of the phenomena in molecular structure which these experiments may serve to explain and illustrate, Philosophical Magazine Series 5, 7:41, 98-108, DOI: $10.1080 / 14786447908639566$

To link to this article: http://dx.doi.org/10.1080/14786447908639566

Published online: 13 May 2009.

Submit your article to this journal $\sqsubset$

山 Article views: 1

Q View related articles ¿ 
3. That the change in the adjustment of the collimator introduced by the curvature of the prism is very small. One prism, which I know to be exceptionally bad, gave a difference of a half per cent. in the focal length of the collimator. It is not the change of focus introduced by the curvatare of the prism which makes the method inaccurate when the prism is bad, but the difference in the change of focus in the two positions of the prism. This is one of the reasons why it is better to take the two positions of the prism not too far away from minimum deviation. The small displacement of the prism will only introduce a small variation in the focal length due to the curvature of the faces.

XIII. On the Morphological Laws of the Configurations formed by Magnets floating vertically and suljected to the Attraction of a superposed Magnet; with Notes on some of the Phenomena in Molecular Structure which these experiments may serve to explain and illustrate. By ALFRED M. MAYER*.

$\mathbf{I}^{\mathrm{N}}$ $\mathrm{N}$ the May Number (1878) of this Journal (page 397 of vol. v.), a short note was published on my experiments with magnets floating vertically and subjected to the attraction of a superposed magnet. The object of this paper is to present accurate diagrams of the configurations formed by the floating magnets, and to give the laws ruling these configurations, with some notices of the peculiarities of these forms. At the same time I will show how neatly these experiments illustrate several phenomena in the molecular structure of matter.

The Diagrams.--'These diagrams show the configurations formed by numbers of magnets extending from two to twenty. They were obtained as follows:- The number of needles forming a configuration were floated in a bowl filled to its brim with water. The eye-ends of the needles, which protruded a short distance beyond the tops of the corks, were of S. polarity. A cylindrical magnet, 38 centims. long and 15 millims. in diameter, was clamped in a vertical position, with its $N$. end at the constant distance of 60 millims. above the tip of the needle, which floated in the line of the axis of the magnet. I tipped the ends of the needles with printer's ink; and when the configuration had formed and was stationary, I brought down upon the needles a piece of flat cardboard, and thus obtained prints from nature. Around each of the dots on the card-

* Communicated by the Author. 
board I drew a black disk. The centres of these disks I joined by lines, in order to bring before the eye the contours of the configurations. After the diagrams of the configurations had been obtained in this way, they were placed at a fixed distance from the camera ; and photo-engravings were thus made, of about one half the sizes of the original prints.

The Morphological Laws of the Configurations.-The configurations male by the floating magnets form well-marked groups or classes, which may be designated in order as primary, secondary, tertiary, quaternary, \&c. The stable configurations of one class form the nuclei to the succeeding ones.

Looking at the diagrams, the reader will see that figures 2 to $8 a$ inclusive form the primaries. Figure $8 b$ begins the secondaries, for it is the hexagon with 2 for nucleus. Configurations $8 b, 8 c, 9,10 b, 10 a, 11,12,13,14,15,16,17,18 a$, $18 b$, and $19 a$ are secondaries, having respectively for nuclei the primaries $2,2,2,2,3,3,4,4,5 a, 6 a, 7,7,7,8 a, 8 a$. The configuration $10 b$ is not found in the diagrams; it is the same as the 10 which forms the nucleus of 20 , only the two central lateral needles are further removed outward from the vertical axis of the figure 10 .

The group of $19 b$ begins the tertiaries. Of these I do not give diagrams, but indicate their structure by giving the numbers of the secondaries forming their nuclei, and then give the numbers of needles grouped around these nuclei. Thus, tho structure of the configuration formed of 47 needles is indicated by $47=(18+14)+15$; which means that 47 needles form a configuration which has 18 for its inner nucleas, surrounded by 14 needles, and these in turn surrounded by 15 ; and as $18+14$ forms the tertiary which is the nucleus to this quaternary 47 , we enclose 18 and 14 in parenthesis.

I here give the configurations to 51 inclusive, which form begins the quinaries.

The configurations of the same number of magnets are lettered $a, b$, c, to indicate their degrees of stability, $a$ being always the most stable form. I have, however, lettered the configuration of 8 magnets in the order of their increasing areas, in order to make them serve better for the purpose of illustrating the phenomena of isomerism. Really $8 c$ is more stable than 88 . 

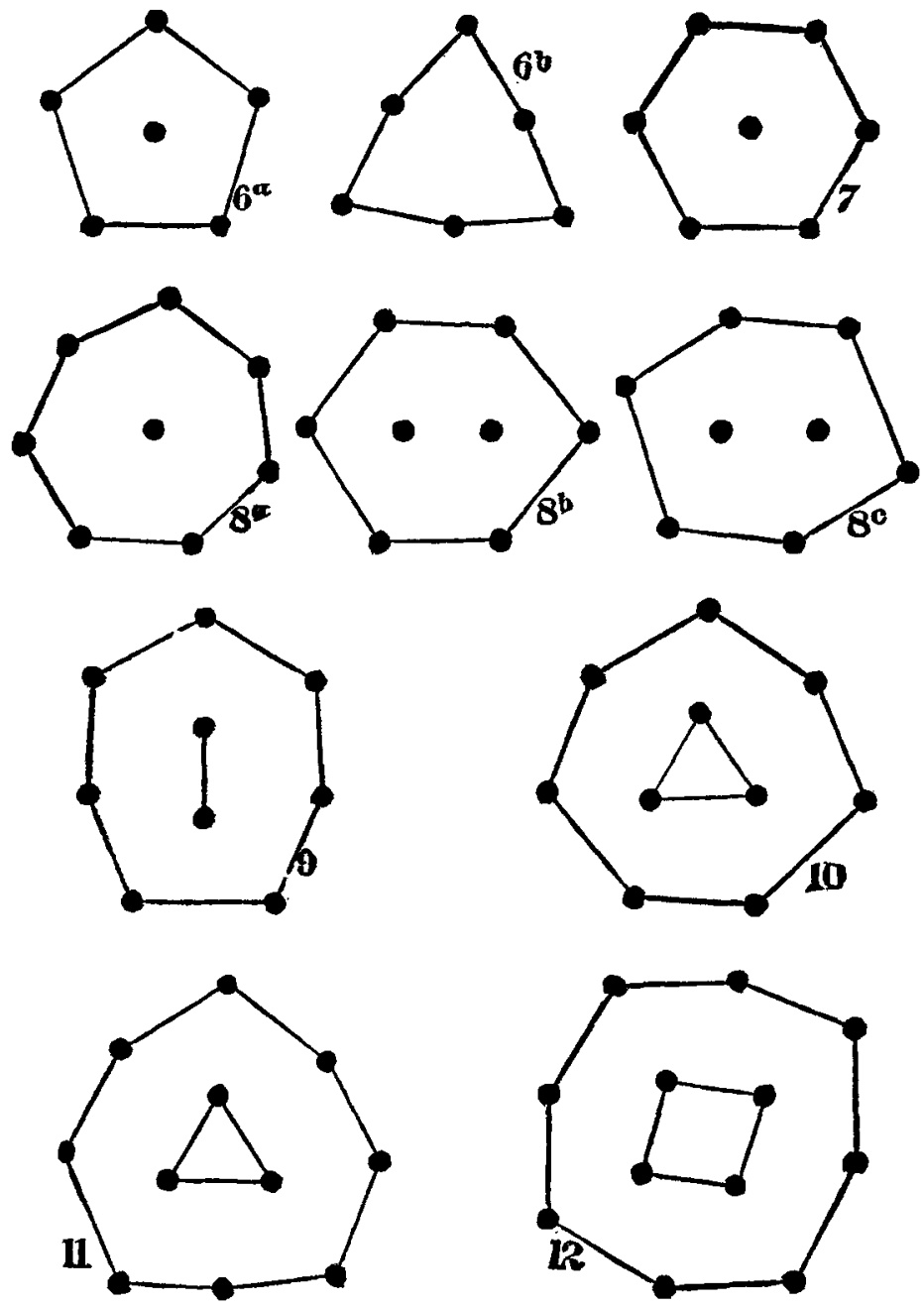
Configurations formed by Floating Magnets.
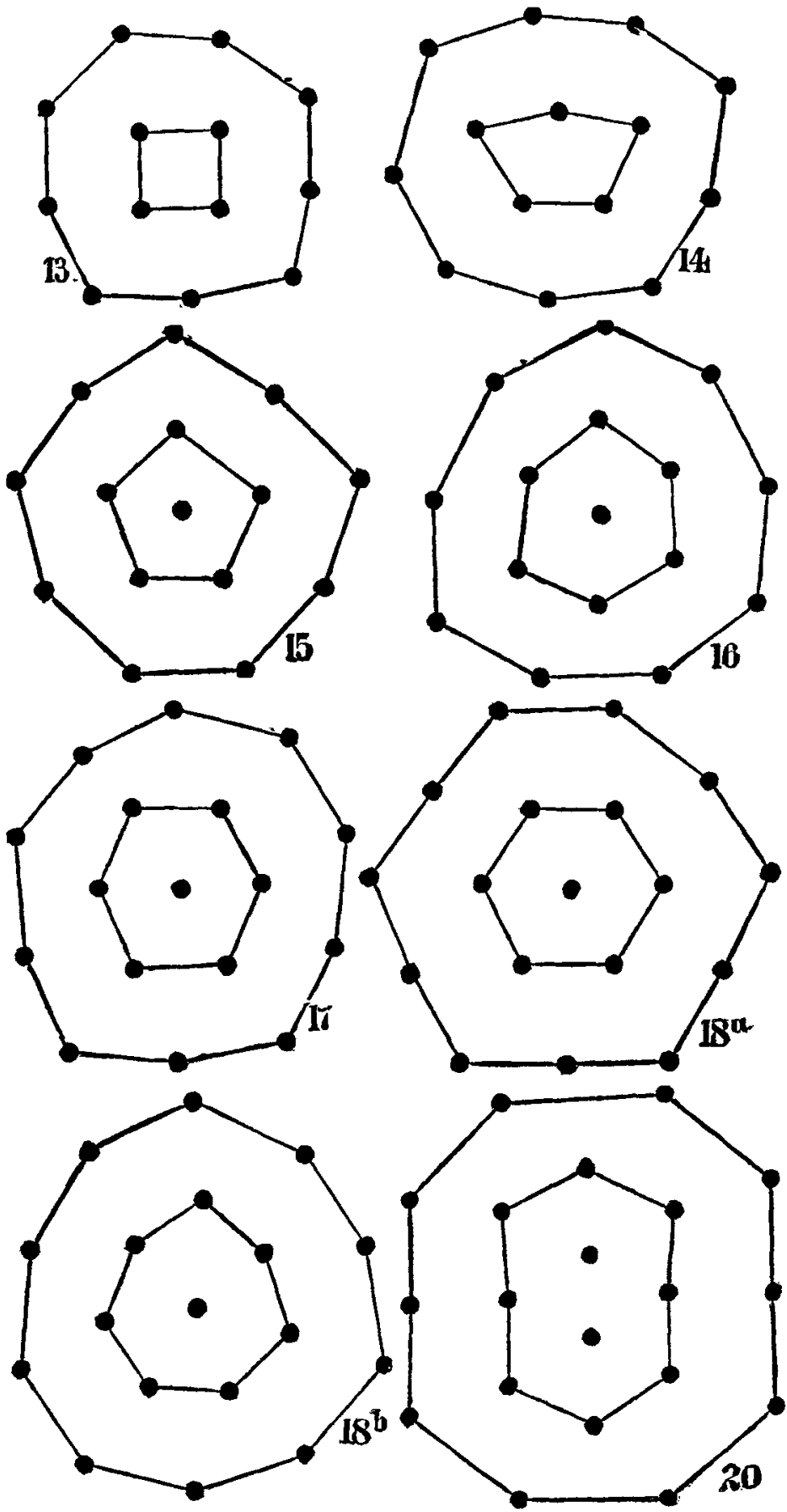
Tertiaries.

$\begin{array}{lll}19 b=9+10 & 25 a=12+13 & 29 a=16+13 \\ 20 a=9+11 & 25 b=13+12 & 29 b=17+12 \\ 20 b=10+10 & 26 a=13+13 & 30 a=17+13 \\ 21 a=10+11 & 26 b=14+12 & 30 b=18+12 \\ 21 b=11+10 & 27 a=14+13 & 31=18+13 \\ 22=11+11 & 27 b=15+12 & 32=18+14 \\ 23=11+12 & 28 a=15+13 & 33=18+15 \\ 24 a=12+12 & 28 b=16+12 & 34 a=(8 a+12)+14 \\ 24 b=11+13 & & \end{array}$

Quaternaries.

$\begin{array}{ll}34 b=(9+10)+15 & 43=(15+14)+14 \\ 35 a=(9+12)+14 & 44=(15+14)+15 \\ 35 b=(10+12)+13 & 45=(16+14)+15 \\ 36=(10+12)+14 & 46=(18+14)+14 \\ 37=(10+13)+14 & 47=(18+14)+15 \\ 38=(11+13)+14 & 48=(18+15)+15 \\ 39=(11+13)+15 & 49=(18+15)+16 \\ 40=(13+13)+14 & 50=(8+11+15)+16 \\ 41=(13+13)+15 & 51 a=(8+12+15)+16 \\ 42=(13+14)+15 & \end{array}$

Quinaries.

$51 b=(9+12+14)+16$.

I do not say that the above list contains all the possible combinations. The list is more for the purpose of establishing the laws which I have already formulated.

In my first publication I gave two configurations for four needles:-one having the needles at the corners of a square, and a stable form; the other unstable, and formed of a triangle containing a central needle. I have concluded that this form does not exist; at least its existence is so transient that it has never remained long enough for me to take a print of it.

I have stated that $19 b$ begins the tertiaries. This is an unstable configuration, and is formed of 9 surrounded by 10 magnets. The other $19,19 a$, is stable, and is formed of $8 a$ surrounded by 11 magnets. It is to be remarked that not alone the tertiaries, but the configurations in the other classes begin with an unstable group of magnets. Thus $8 \mathrm{e}$ begins the secondaries, $19 b$ the tertiaries, $34 b$ the quaternaries, and $51 b$ the quinaries.

The reader has seen that a given number of magnets may form two or more different configurations. Thus fire magnets form two, $5 b$ a square with a magnet at its centre, and 
$5 a$ a pentagon. Six magnets give $6 a$ and $6 b$. With eight magnets we obtain three configurations, $8 a, 8 b$, and $8 c$. Now the different configurations formed of the same number of magnets always exhibit different degrees of stability. Vibration of the less stable forms (produced by alternately lifting and lowering the superposed magnet) sends them into the stable forms. Thus, $5 b$ on vibration rearranges itself into $5 a$, $6 b$ into $6 a$, and $8 c$ or $8 b$ into $8 a$. With the configurations of higher classes (the tertiaries, quaternaries, \&c.), even a knock on the table is sufficient to cause the needles of the unstable configuration to move to positions of stable equilibrium.

On looking at the diagrams, it will be observed that only the stalle primaries form the nuclei of the secondaries; and, moreover, those primaries which are not dimorphons, like 2, 3,4 , and 7 , serve as nuclei to more than one secondary. Thus, 2 is the nucleus of $8 a, 8 b, 8 c, 9$, and $10 b ; 3$ is the nucleus of $10 a$ and 11 ; and 7 is the nucleus to 16,17 , and 18 ; while each of the other stable and dimorphous primaries, $5 a, 6 a$, and $8 a$, appears only once as nucleus, respectively to 14,15 , and 18b. This same power of the most stable nuclei to resist outside stress is shown in the configurations of the tertiary and quaternary classes, where the secondary 11 appears as nucleus to $21,22,23$, and 24 . The secondary $18 a$ persists in even a more marked manner as a nucleus. This 18 a has the contour of that very stable 7 (the only configuration possible with 7 magnets) which forms its nucleus. Among the tertiaries $18 \mathrm{a}$ is the nucleus of $30 b, 31,32$, and 33 ; while in the quaternaries it forms the inner nucleus of $46,47,48$, and 49 . The fact of the persistence of these stable forms as nuclei may be suggestive to chemists and crystallographers.

It is here to be remarked that (as a general rule holding good in all the classes), of two configurations made up of the same number of magnets, that configuration is the more stable which has the least number of needles for its nucleus.

Illustrations of Molecular Structure. (1) Unstable Molecular Equilibrium.-That the molecules in a body may be in a state of unstable equilibrium so delicately balanced that a slight extraneous action of pressure, heat, light, \&c. may cause a new molecular arrangement in the body, is shown in many facts. A few of the more familiar ones will answer for our purpose. Thus quiet water, which remains liquid at a temperature of $10^{\circ} \mathrm{C}$. or more below $0^{\circ} \mathrm{C}$., changes suddenly into ice when agitated; and during this solidifieation its temperature rises. In like manner a supersaturated solution of disodium sulphate solidifies when a crystal of this substance is dropped into it. Another instance of a sudden change from 
an unstable to a stable molecular condition is shown when the yellow crystals of mercuric iodide change, on the touch of a glass rod, to a scarlet colour, with a perceptible motion of their particles. These and similar phenomena are illustrated by the change of unstable to stable configurations caused by vibration, shock, and varying conditions of stress. Thus $5 b$ changes into $5 a, 6 b$ into $6 a$, and $8 c$ and $8 b$ into $8 a$.

(2) Illustrations of Expansion on Solidification, as shown by water, bismuth, antimony, cast iron, \&c., are readily given by the floating magnets. One volume of water at $0^{\circ} \mathrm{C}$. expands, on freezing, into about one and one tenth volume of ice. It happens that the area of $5 b$ is greater than the area of $5 a$ by about one tenth; so that the increase in area which takes place when the pentagon of $5 a$ is changed into the square $5 b$ may represent the increase in the volume of water when it changes into ice.

It will be observed, on an examination of the diagrams, that, of two configurations formed of the same number of needles, that configuration which has the larger area has a magnet in its centre. Thus $5 b$ exceeds in area $5 a$, and $6 a$ is of greater area than $6 z$. To see the effect of a repulsive centre on a configuration, compare the areas of the two squares 4 and $5 b$, and of the two pentagons $5 a$ and $6 a$. The most marked effect of a repulsive central magnet is seen on comparing 14 with 15 . The outside contour of each is formed of 9 magnets. The nucleus of 14 is the peculiar flattened pentagon, which is expanded into symmetry on the addition of another magnet, while at the same time the outside contour of 15 conforms to the regular pentagonal nucleus. These phenomena are so suggestive, that I make bold to put the quesion, May it not be that there is an actual centralization of atoms in the molecule when a body expands in solidifying, and in the case where of two or more isomeric bodies one has always the minimum density? I offer this as a suggestion which may be worthy of the consideration of crystallographers.

(3) Illustrations of Allotropy and Isomerism.-The most interesting of our experiments with the floating magnets are those illustrating the phenomena of allotropy and isomerism. It is well known that an elementary substance may exist under very different forms. By the action of heat, electricity, \&c. an element may have its physical and chemical properties so changed that no one would suppose that the different bodies thus made out of one and the same element were really all of the same substance. Yet the body remains elementary under the different appearances; for it is impossible by any means of subtraction to get any thing but the elementary substance 
from it. Phosphorus, sulphur, and carbon give instances of allotropy. Thus graphite and the diamond are both carbon; yet how different are they! One is soft, opaque, black, and with a metallic lustre; the other is the hardest of bodies, transparent, and resplendent by its refractive action on light. Graphite is a good conductor of electricity, crystallizes in small six-sided tables which belong either to the hexagonal or monoclinic system, and has a specific gravity of $2 \cdot 2$; while the diamond is a bad conductor of electricity, crystallizes in the monometric system, and has a specific gravity of 3.5 . Whenever an element or a compound takes two different crystal-forms, these different crystals always differ in their density.

These differences of form and density shown in allotropy and isomerism are well illustrated in the configurations which are formed of the same number of magnets. Take figures $5 a$ and $5 b$. The first is a pentagon; the second is a square with a magnet in its centre. The forces in these floating magnets and in the superposed magnet remain the same in all the configurations; and these have all been printed from needles floated in water whose surface was at a constant perpendicular distance from the pole of the superposed magnet. Thus we see how the same atoms, endowed with forces of the same strength, may take different relative positions, and thus produce very different crystal-forms in the same matter. We may take $5 a$ for an illustration of the atomic arrangement in the diamond, while 52 may stand for graphite. But there is always a change of density accompanying the different forms in allotropy; and this fact is also illustrated by configurations $5 a$ and $5 b$. In bodies formed of the same kind of elementary atoms, as in allotropy, it is evident that their relative densities will be directly as the number of atoms contained in the unit of volume. As our configurations illustrating allotropy contain the same number of magnets, it follows that the relative densities of these configurations are inversely as their areas. Now the area of $5 a$ (measured on the original prints) is 818 square millimetres, and the area of $5 b$ is 992 square millimetres; hence the density of $5 a$ is to the density of $5 b$ as 992 is to 818. Thus we see how the arrangement of magnets in 5a may stand for the molecular structure in the diamond, while $5 b$ may stand for that in graphite.

Numerous instances exist in chemistry of the same elements combined in the same proportions, yet producing bodies crystallizing in different forms, and having different densities, colour, transparency, hardness, \&c. As examples of this phenomenon of isomerism we may cite calcium carbonate, which 
crystallizes in two forms, differing in density-viz. as calc spar, with a specific gravity of $2 \cdot 72$, and as arragonite, with a specific gravity of 2.93 . Configuration $6 a$ may stand for the molecular structure of calc spar, while $6 b$ may stand for that of arragonite. The relative densities of these two configurations are as 208 to 247 .

A striking example of isomerism is given in titanic acid, which crystallizes in three distinct forms :-as anatase, specific gravity 3.82 ; as Brookite, specific gravity 4.02 ; and as rutile, specific gravity $4 \cdot 25$. These three isomers may be illustrated by $8 c, 8 b$, and $8 a$, which have respectively the densities of 382,364 , and 360 .

It will, of course, be understood that the above parallelisms are given merely as illustrations of how our experiments may serve to explain and illustrate the phenomena, on the assumption of the atomic hypothesis, and on the supposition that the actions which, in the experiments, take place in a plane, may similarly take place among repelling and attracting points situate in space of three dimensions.

Other forms of the Experiments.-Instead of floating the magnets, they may be suspended by fine silk fibres. In this method of experimenting the attractive action of the superposed magnet is replaced by the action of gravity, which draws the mutually repellant needles towards the vertical.

An advantage of this form of the experiment is that the configuration can be transported, and may thus serve in illustration of a moving molecule as set forth in the kinetic theory of gases. It is interesting to watch the mutual actions of two or more approaching configurations, and to observe the motions in the exterior and in the contour of a suspended configuration on its impact against a resisting or a yielding surface.

Professor 0. N. Rood suggested to me to replace the suspended magnets by gilded pith balls, hung by silk fibres and similarly electrified.

Professor Frederick Guthrie, under date of May 21, writes:- "If the corks are made somewhat wider than in your larger needles, the needles move and arrange themselves very quickly if they are turned over and floated on perfectly pure and freshly filtered mercury. Those which reach the edge incline with their corks in the capillary trough."

Method of projecting the magnified Images of the experiments on a Screen.-To exhibit these experiments before a large audience it is best to use short magnets made as follows :Magnetize rather large sewing-needles, with their points all of the same polarity; then take each needle between the flat jaws 
of a pair of pliers, and with a pair of cutting-pliers snap off the needle close to the jaws of the other pliers. Thus form a series of magnets about ${ }_{4}^{3}$ inch in length. Run each of these through a thin section of a small cork, and then coat both needle and cork with shellac varnish. Float these magnets in a glass tank placed over the condensing lens of a verticallantern ; or you may even float them directly on the condenser itself, if this is made of an inverted glass shade filled with water. This form of condenser was first used by Dr. R. M. Ferguson, of Edinburgh.

Figure 1 shows the arrangement of the experiment. The rays of light, $R$, from a heliostat, or from an oxyhydrogen light,

1.

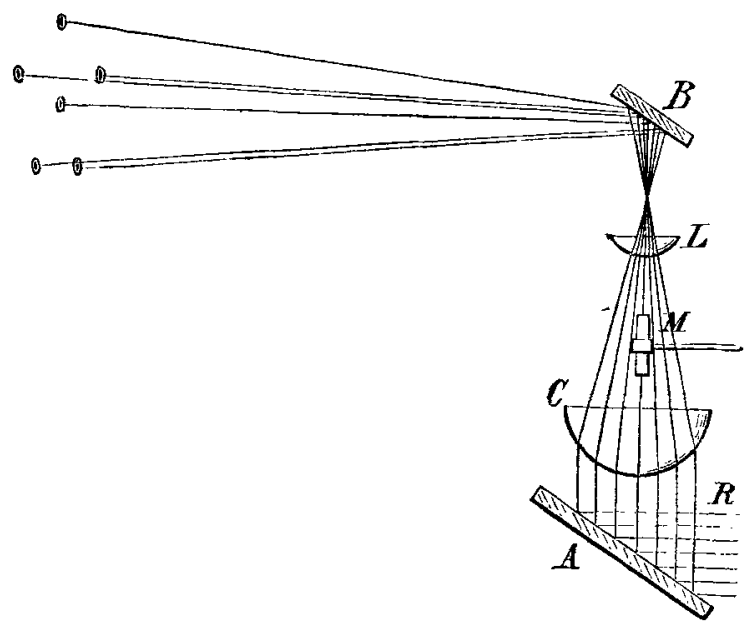

fall on an inclined mirror, A, placed under the water condenser, C. The needles float on the surface of the water in this condenser. The rays which have passed through the lens, $\mathrm{L}$, are reflected by the swinging mirror, $\mathrm{B}$, to the distant screen, where they form the images of the floating magnets. The magnet is held over the needles at $M$ by means of a wire which is wrapped round the magnet to serve as a handle. If a long magnet be used, it will work well if its pole is brought over the needles* by inclining it.

These experiments with floating magnets give foreible presentations of the reign of law. It is indeed quite impressive to see order being evolved out of chaos as we hold a magnet

* The magnetic needles in the experiments may be replaced by pieces of soft iron wire, which will he magnetized by the induction of the superposed magnet. 
over a number of needles carelessly thrown on water, and witness them approaching and, one after the other, entering into the structure of that geometric figure which conforms to the number of magnets composing it.

XIV. A Condenser of Variable Capacity, and a Total-Reflexion Experiment. By C. V. Boys, A.R.S.M., Lecturer for the Term on Natural Science at Uppingham School*.

WISHING to show my pupils the effect of condensation on the spark, I thought a condenser the capacity of which could be reduced gradually to nothing would be most suitable. So I made this simple contrivance, which answered its purpose well :-

$A$ glass tube is sealed at one end and is covered with tinfoil for one third of its length ; this forms the outer coating. The inner coating consists of a test-tube with the rim cut off, also covered with tinfoil ; this is fixed to a wire, and can be drawn in and out. When it is fully in, the condenser has its maximum capacity; when drawn out as far as possible, the two coatings are too far apart to have any sensible action, and the capacity is zero.

On hanging this on the conductor of a Holtz machine the effect on the spark is well shown. Let the wire be first pushed in as far as possible, the condenser then acts to its full extent; but on gradually drawing it out the sparks are less and less bright, but follow one another more and more rapidly, till at last, when it is fully out, they have passed gradually to the almost continuous pale spark so characteristic of a Holtz machine. To show the effect best, the poles should not be more than about half an inch apart. Of course much ozone is formed inside the tube.

The total-reflexion experiment was an accident. A small condenser made of a test-tube gave way under the strain, a minute hole being pierced in the bottom, through which sparks passed almost continually. No light could be seen anywhere except on the rim of the tube, which formed a brilliant circle of light. The light from the spark was totally internally reflected in the substance of the tube till it reached the rim, which it struck normally. The bright circle of light (the tube itself being dark) was very striking; and the experiment is a far truer illustration of total internal reflexion than the more beautiful one with a stream of water. The tube is, unfortunately, broken; and I have not succeeded in piercing another with the spark. A crack made with a hot wire does notdoso well.

* Communicated by the Physical Society. 